\title{
ASYMPTOTIC EQUIVALENCE OF IMPULSIVE DIFFERENTIAL EQUATIONS IN A BANACH SPACE
}

\author{
D.D. Bainov, S.I. Kostadinov and A.D. MyshKis
}

\begin{abstract}
By means of Schauder's fixed point theorem sufficient conditions for asymptotic equivalence of impulsive equations in a Banach space are found.
\end{abstract}

\section{Introduction}

Impulsive differential equations are a powerful apparatus for mathematical simulation in physics, chemical technology, ecology, biotechnologies, impulse technology and industrial robotics. That is why the theory of impulsive differential equations develops actively in the last 20 years [4], [8-13], [15]. The presence of phenomena specific for the impulsive differential equations such as beating of the solutions, merging of the solutions, bifurcation, dying of the solutions, loss of the property of autonomy, etc. makes this theory richer than the theory of ordinary differential equations and, on the other hand, in the investigation of impulsive differential equations a prefiminary modification of the methods used is necessary.

In relation to the mathematical simuiation in theoretical physics recently the investigation of impulsive differential equations in abstract spaces began [1], [2]. In the present paper the asymptotic equivalence of impulsive differential equations in a Banach space is investigated. We shall note that the asymptotic equivalence of systems of ordinary differential equations has been investigated by many authors [3], [5-7], [14], [16].

The present investigation is supported by the Ministry of Culture, Science and Education of People's Republic of Bulgaria under Grant 61. 


\section{Preliminary notes}

Consider the impulsive equations

$$
\begin{gathered}
\frac{d x}{d t}=A(t) x\left(t \notin\left\{t_{j}\right\}_{j=1}^{\infty}\right) \\
x\left(t_{j}^{+}\right)=L, x\left(t_{j}^{-}\right)(j=1,2,3, \ldots)
\end{gathered}
$$

and

$$
\begin{gathered}
\frac{d y}{d t}=A(t) y+f(t, y)\left(t \notin\left\{t_{j}\right\}_{j=1}^{\infty}\right) \\
y\left(t_{j}^{+}\right)=\left(L_{j}+H_{j}\right) y\left(t_{j}^{-}\right)(j=1,2,3, \ldots)
\end{gathered}
$$

in a real or complex Banach space $X$. Here $t$ varies in the interval $[t, \infty) ; A(t)$ : $X \rightarrow X$ (for each $t \in[t, \infty)$ ) is a linear bounded operator and the operatorvalued function $A(\cdot)$ is continuously continuable from $\left(t_{j-1}, t_{j}\right)$ to $\left[t_{j-1}, t_{j}\right](j=$ $\left.1,2,3, \ldots, t_{0}=\bar{t}\right) ; L_{j}: X \rightarrow X(j=1,2,3, \ldots)$ are lincar bounded operators having bounded inverse ones and $L_{j} X=X$; the function $f:[t, \infty) \times X \rightarrow X$ is continuosiy continuable from $\left(t_{j-1}, t_{j}\right) \times X$ to $\left[t_{j-1}, t_{j}\right] \times X(j=1,2,3, \ldots) ; H_{j}$ : $X \rightarrow X(j=1,2,3, \ldots)$; the sequence $T=\left\{t_{j}\right\}_{j=1}^{\infty}$ satisfies the condition

$$
\bar{t}<t_{1}<t_{2}<\cdots \rightarrow \infty
$$

We assume that the solutions of equations (1), (2), (3) and (4) are continuous from the left, thus in the conditions of a jump (2) and (4) instead of $\vec{t}_{j}$ we shall write $t_{j}$. If $x$ is a solution of equation (1), (2) on the interval $[a, \infty)$, we shall write $x \in((1),(2))_{\alpha}$ and analogously of equation (3), (4). An arbitrary solution of impulsive equation (1), (2) defined on some subinterval of $[\bar{t}, \alpha)$ is continuable to the whole interval which for impulsive equation (3), (4) in general is not true.

Denote by $W(t, \tau)(\bar{t} \leq t, \tau<\infty)$ the evolutionary operator of impulsive equation (1), (2) associating with each elcment $x_{0} \in X$ the solution $x(t)=$ $W(t, \tau) x_{0}$ of $(1),(2)$ with condition $x(\tau)=x_{0}$.

The following equalities are valid

$$
W_{i}^{\prime}(t, \tau)=A(t) W(t, \tau)\left(t \notin\left\{t_{j}\right\}_{j=1}^{\infty}\right), W\left(t_{j}^{+}, \tau\right)=L_{j} W\left(t_{j}, \tau\right)
$$

Assume that the space $X$ is split up in a direct sum of the subspaces $X_{1}$ and $X_{2}$, i.e. $X=X_{1}+X_{2}$ and let $P_{1}, P_{2}$ be the corresponding complementary projectors $\left(P_{1}+P_{2}=I\right.$, where $I$ is the identity operator $)$. Set $W_{i}\left(t_{1}, t_{2}\right)=$ $W\left(t_{1}\right) P_{i} W^{-1}\left(t_{2}\right)(i=1,2)$, where $W(t)=W(t, \bar{t})$.

Let $K \subseteq[\bar{t}, \infty)$. Denote by $S(K, X)$ the Banach space of all bounded functions $x: K \rightarrow X$ which are continuous for $t \neq t ; \in K$ continuous from the left, have a limit from the right, provided with the norm $\||x|\| \mid=\sup _{t \in K}\|x(t)\|$. 
Lemma 1. Let $F \subset S([\alpha, \infty), X)(\alpha \geq \bar{t})$ and let the following conditions hold:

1. There exists a function $\varphi_{0} \in S([\alpha, \infty), X)$ such that $\left\|\varphi(t)-\varphi_{0}(t)\right\| \underset{t \rightarrow \infty}{\rightarrow} 0$ uniformly with respect to $\varphi \in F$.

2. The set $F$ is equicontinuous on each interval $[\alpha, \infty) \cap\left(t_{j-1}, t_{j}\right](j=$ $1,2,3, \ldots)$.

3. For each $t \in[\alpha, \infty)$ the set $\{\varphi(t): \varphi \in F\}$ is relatively compact in $X$.

Then $F$ is relatively compact in $S([\alpha, \infty), X)$.

Lemma 1 is proved in a standard way by the construction for any $\varepsilon>0$ of an $\varepsilon$-lattice for $F$ in the space $S([\alpha, \infty), X)$.

\section{Main results}

Set $h_{j}=L_{j}^{-1} H_{j}$ and $x_{j}=x\left(t_{j}\right)$ where $x(t)$ is an arbitrary function of $t$.

Definition 1 . The impulsive equations (1), (2) and (3), (4) are called asymptotically equivalent if for any bounded solution $x \in\left((1),(2)_{i}\right.$ there exists $\alpha \in[t, \infty)$ and a solution $y \in((3),(4))_{\alpha}$ such that $y(t)-x(t) \underset{t \rightarrow \infty}{\rightarrow} 0$ and, conversely, for any bounded solution $y \in((3),(4))_{t}$ there exists a solution $x \in((1),(2))_{\bar{t}}$ for which $y(t)-x(t) \underset{t \rightarrow \infty}{\rightarrow} 0$.

Theorem 1. Let the following conditions hold:

1. There exists a bounded solution $x \in((1),(2))_{\bar{t}}$.

2. There exists a constant $R>0$ for which

(5) $\int_{t}^{t} \sup _{z}^{\prime}\left\|W_{1}(t, s) f(s, z)\right\| d s+\sum_{i<t_{j}<t} \sup _{z}^{\prime \prime}\left\|W_{1}\left(t, t_{j}\right) h_{j}(z)\right\|+$ $+\sup _{i \leq r \leq t}\left[\int_{t}^{\infty} \sup _{z}^{\prime}\left\|W_{2}(\tau, s) f(s, z)\right\| d s+\sum_{t \leq t_{j}<\infty} \sup _{z}^{\prime \prime}\left\|W_{2}\left(\tau, t_{j}\right) h_{j}(z)\right\|\right] \underset{t \rightarrow \infty}{\rightarrow} 0$

where $\sup _{z}^{\prime}$ is taken over the set $\{z \in X:\|z-x(s)\| \leq R\}$ and $\sup _{z}^{\prime \prime}$ over the set $\{z \in X:\|z-x\| \leq R$,$\} .$

3. The set $f\{(t, z): \bar{t} \leq t<\infty,\|z-x(t)\| \leq R\}$ is relatively compect in $X$.

4. For $j=1,2,3, \ldots$ the mappings $H_{j}$ are continuous on the balls $B_{j}=$ $\left\{z \in X:\left\|z-x_{j}\right\| \leq R\right\}$ and the set $\bigcup_{j=1}^{\infty} h_{j}\left(B_{j}\right)$ is relatively compact in $X$.

Then for some $\alpha \in[\bar{t}, \infty)$ there exists a solution $y \in\left((3,(4))_{\alpha}\right.$ for which $y(t)-x(t) \underset{t \rightarrow \infty}{\rightarrow} 0$. If, moreover, the left-hand side of the relation in (5) (as a 
function of $t$ ) belongs to the space $L_{p}(\bar{t}, \infty)$ for some $p \in[1, \infty]$, then $\| y(t)-$ $x(t) \| \in L_{p}(\alpha, \infty)$.

Proof: Denote the left-hand side of relation (5) by $F(t)$ and choose $\alpha \geq \bar{t}$ so large that $F(t) \leq R$ for $t \geq \alpha$. The set $D=\{z \in S([\alpha, \infty), X): \| z-$ $\left.\left.x\right|_{[a, \infty)} \| l \leq R\right\}$ is nonempty, convex, closed and bounded.

On $D$ define the operator $Q$ by means of the formula

$$
\begin{aligned}
(Q z)(t)=x(t)+\int_{\alpha}^{t} W_{1}(t, s) f(s, z(s)) d s+\sum_{\alpha \leq t_{j}<t} W_{1}\left(t, t_{j}\right) h_{j}\left(z_{j}\right)- \\
-\int_{t}^{\infty} W_{2}(t, s) f(s, z(s)) d s-\sum_{t \leq t_{j}<\infty} W_{2}\left(t, t_{j}\right) h_{j}\left(z_{j}\right)
\end{aligned}
$$

where $z \in D, t \in[\alpha, \infty)$. The convergence of the improper integral and the series in $(6)$ follws from the fact that $F(t)<\infty$.

We shall show that the operator $Q$ satisfies the conditions of Schauder's fixed point theorem.

1. $Q: D \rightarrow D$. In fact, from the uniform convergence of the improper integral and the series in the right-hand side of equality (6) and from the choice of $\alpha$ it follows that $Q z \in S([\alpha, \infty), X)$ and that $\|(Q z)(t)-x(t)\| \leq R, \alpha \leq t<$ $\infty$.

2. The operator $Q$ is continuous. In fact, let $\varepsilon>0$ be an arbitrarily chosen number and let $\xi \geq \alpha$ be choosen so that $F(s)<\frac{\varepsilon}{8}$ for $s \geq \xi$. Let $z, z_{1} \in D$ and $\alpha \leq t \leq \xi$. Then

$$
\begin{aligned}
\left\|(Q z)(t)-\left(Q z_{1}\right)(t)\right\| & \leq \int_{\alpha}^{t}\left\|W_{1}(t, s)\left[f(s, z(s))-f\left(s, z_{1}(s)\right)\right]\right\| d s+ \\
& +\int_{t}^{\xi}\left\|W_{2}(t, s)\left[f(s, z(s))-f\left(s, z_{1}(s)\right)\right]\right\| d s+ \\
& +\sum_{\alpha \leq t_{j}<t}\left\|W_{1}\left(t, t_{j}\right)\left[h_{j}\left(z_{j}\right)-h_{j}\left(z_{1 j}\right)\right]\right\|+ \\
& +\sum_{t \leq t_{j}<\xi}\left\|W_{2}\left(t, t_{j}\right)\left[h_{j}\left(z_{j}\right)-h_{j}\left(z_{1 j}\right)\right]\right\|+ \\
& +\int_{\xi}^{\infty}\left\|W_{2}(t, s)\left[f(s, z(s))-f\left(s, z_{1}(s)\right)\right]\right\| d s+ \\
& +\sum_{\xi \leq t_{j}<\infty}\left\|W_{2}\left(t, t_{j}\right)\left[h_{j}\left(z_{j}\right)-h_{j}\left(z_{1 j}\right)\right]\right\|
\end{aligned}
$$

Due to the continuity of the functions $f$ and $h_{j}$ for a fixed function $z_{1}$ a number $\eta>0$ can be found such that for $\left\|\left|z-z_{1} \|\right|<\eta\right.$ each of the first four addends in the right-hand side of the last inequality should be smaller than 
$\frac{\varepsilon}{8}$. But then the right-hand side of the last inequality becomes smaller than $4 \frac{\varepsilon}{8}+2 \frac{\varepsilon}{8}+2 \frac{\varepsilon}{8}=\varepsilon$. For $t>\xi$ the following inequalities are valid

$$
\left\|(Q z)(t)-\left(Q z_{1}\right)(t)\right\| \leq\|(Q z)(t)-x(t)\|+\left\|\left(Q z_{1}\right)(t)-x(t)\right\|<2 \frac{\varepsilon}{8}<\varepsilon
$$

Hence the operator $Q$ is continuous.

3. We shall show that the set $Q D$ is relatively compact in $D$. For this purpose we shall show that the conditions of Lemma 1 are fulfilled for $F=$ $Q D, \varphi_{0}=x$. Condition 1 of Lemma 1 follows immediately from relation (5).

We shall show that condition 2 of Lemma 1 holds. Let $z \in D$ and $t^{\prime}, t^{\prime \prime} \in$ $\left(t_{k-1}, t_{k}\right)$ for some $K$ where $t^{\prime} \leq t^{\prime \prime}$. Then, after elementary transformations we obtain the equality

$$
\begin{aligned}
(Q z)\left(t^{\prime}\right)-(Q z)\left(t^{\prime \prime}\right)= & x\left(t^{\prime}\right)-x\left(t^{\prime \prime}\right)-\int_{t^{\prime}}^{t^{\prime \prime}} W_{1}\left(t^{\prime \prime}, s\right) f(s, z(s)) d s- \\
& -\int_{\alpha}^{t^{\prime}}\left[W_{1}\left(t^{\prime \prime}, s\right)-W_{1}\left(t^{\prime}, s\right)\right] f(s, z(s)) d s+ \\
& +\sum_{\alpha \leq t_{j}<t_{k}}\left[W_{1}\left(t^{\prime}, t_{j}\right)-W_{1}\left(t^{\prime \prime}, t_{j}\right)\right] h_{j}\left(z_{j}\right)- \\
& -\int_{t^{\prime}}^{t^{\prime \prime}} W_{2}\left(t^{\prime}, s\right) f(s, z(s)) d s- \\
& -\int_{t^{\prime \prime}}^{\infty}\left[W_{2}\left(t^{\prime}, s\right)-W_{2}\left(t^{\prime \prime}, s\right)\right] f(s, z(s)) d s- \\
& -\sum_{j=k}^{\infty}\left[W_{2}\left(t^{\prime}, t_{j}\right)-W_{2}\left(t^{\prime \prime}, t_{j}\right)\right] h_{j}\left(z_{j}\right),
\end{aligned}
$$

from which we deduce the estimate

$$
\begin{aligned}
\left\|(Q z)\left(t^{\prime}\right)-(Q z)\left(t^{\prime \prime}\right)\right\| & \leq\left\|x\left(t^{\prime}\right)-x\left(t^{\prime \prime}\right)\right\|+\int_{t^{\prime}}^{t^{\prime \prime}}\left\|W_{1}\left(t^{\prime \prime}, s\right) f(s, z(s))\right\| d s+ \\
& +\int_{\alpha}^{t^{\prime}}\left\|W\left(t^{\prime}\right)-W\left(t^{\prime \prime}\right)\right\| \cdot\left\|P_{1} W^{-1}(s) f(s, z(s))\right\| d s+ \\
& +\sum_{\alpha \leq t_{j}<t_{k}}\left\|W\left(t^{\prime}\right)-W\left(t^{\prime \prime}\right)\right\|\left\|P_{1} W^{-1}\left(t_{j}\right) h_{j}\left(z_{j}\right)\right\|+ \\
& +\int_{t^{\prime}}^{t^{\prime \prime}}\left\|W_{2}\left(t^{\prime}, s\right) f(s, z(s))\right\| d s+ \\
& \left.+\int_{t^{\prime \prime}}^{\infty} \| W\left(t^{\prime}\right)-W\left(t^{\prime \prime}\right)\right] P_{2} W^{-1}(s) f(s, z(s)) \| d s+ \\
& \left.+\sum_{j=k}^{\infty} \| W\left(t^{\prime}\right)-W\left(t^{\prime \prime}\right)\right] P_{2} W^{-1}\left(t_{j}\right) h_{j}\left(z_{j}\right) \|
\end{aligned}
$$


Denote by $I_{1}, \ldots, I_{7}$ the successive addends in the right-hand side of the last inequality and assume that $t^{\prime \prime}-t^{\prime} \rightarrow 0$. Then $I_{1} \rightarrow 0$ since $x \in S([\bar{t}, \infty), X)$. $I_{2}, I_{5} \rightarrow 0$ because of the uniform (with respect to $z, t^{\prime}, t^{\prime \prime}$ ) boundedness of the integrand. $I_{3} \rightarrow 0$ and $I_{4} \rightarrow 0$ since under the signs of the integral and of the sum the first factor tends uniformly to zero and the second one is uniformly bounded. Represent $I_{6}$ for $t \geq t_{k}$ in the form

$$
I_{6}=\int_{t^{\prime \prime}}^{t}+\int_{t}^{\infty} \leq \int_{t^{\prime \prime}}^{t}\left\|W\left(t^{\prime}\right)-W\left(t^{\prime \prime}\right)\right\| \cdot\left\|P_{2} W^{-1}(s) f(s, z(s))\right\| d s+2 F(t)
$$

The second addend in the last inequality can be made arbitrarily small for a sufficiently large value of $t$. For $t$ fixed and $t^{\prime \prime}-t^{t}$ small enough the first addend can also be made uniformly arbitrarily small (analogously to $I_{3}$ ). Hence $I_{6} \rightarrow 0$. Analogously it is proved that $I_{7} \rightarrow 0$.

Thus the equicontinuity of the set $Q D$ on the interval $\left(t_{k-1}, t_{k}\right)$ is proved.

In order to verify condition 3 of Lemma 1 , first we shall show that for $t \in$ $[\alpha, \infty)$ the set $\left\{\int_{t}^{\infty} W_{2}(t, s) f(s, z(s)) d s: z \in D\right\}$ is relatively compact in $X$. Let $\varepsilon>0$ be arbitrarily chosen. Choose $\xi \geq t$ so that $\left\|\int_{\xi}^{\infty} W_{2}(t, s) f(s, z(s)) d s\right\| \leq$ $\frac{\varepsilon}{3}$ for all $z \in D$. The set $\left\{W^{-1}(s) f(s, z(s)): t \leq s \leq \xi, z \in D\right\}$ is relatively compact in $X$. Hence the sets $\left\{\int_{t}^{\xi} W^{-1}(s) f(s, z(s)) d s: z \in D\right\}$ and $\left\{\int_{t}^{\xi} W_{2}(t, s) f(s, z(s)) d s: z \in D\right\}$ are also relatively compact subsets of $X$. For the last set we choose a finite $\frac{\varepsilon}{3}$-lattice- $\left\{\int_{2}^{\xi} W_{2}(t, s) f\left(s, z_{i}(s) d s: i=\right.\right.$ $\left.1, \ldots, N_{i} z_{i} \in D\right\}$ and obtain that the set $\left\{\int_{t}^{\infty} W_{2}(t, s) f\left(s, z_{i}(s)\right) d s: i=\right.$ $1, \ldots, N\}$ forms an $\varepsilon$-lattice for the set $\left\{\int_{t}^{\infty} W_{2}(t, s) f(s, z(s)) d s: z \in D\right\}$ which is therefore relatively compact in $X$.

Analogously the relative compactness in $X$ of the remaining addends is verified, hence of the sum in the right-hand side of equality (6) for arbitrary fixed $t \in[\alpha, \infty)$ as well when $z$ runs over the set $D$. This crables us to apply Lemma 1.

From Schauder's theorem it follows that the operator $Q$ has a fixed point $y \in D$. We shall show that $y \in((3),(4))_{\alpha}$.

Let $t \in[\alpha, \infty) \backslash\{t,\}_{j=1}^{\infty}$. Then, in view of the fact that $x$ is a solution of impulsive equation (1), (2) and of the equality $W_{1}(t, t)+W_{2}(t, t)=I$, we obtain

$$
\begin{aligned}
& y^{\prime}(t)=(Q y)^{\prime}(t)= \\
& =x^{\prime}(t)+W_{1}(t, t) f(t, y(t))+A(t) \int_{\alpha}^{t} W_{1}(t, s) f(s, y(s)) d s+ \\
& \quad+A(t) \sum_{\alpha \leq t_{j}<t} W_{1}\left(t, t_{j}\right) h_{j}\left(y_{j}\right)+W_{2}(t, t) f(t, y(t))- \\
& -A(t) \int_{t}^{\infty} W_{2}(t, s) f(s, y(s)) d s-A(t) \sum_{i<t_{j}<\infty} W_{2}\left(t, t_{j}\right) h_{j}\left(y_{j}\right)= \\
& =A(t) y(t)+f(t, y(t))
\end{aligned}
$$


Let $t=t_{k} \in[\alpha, \infty)$. Then

(8)

$$
\begin{aligned}
& y\left(t_{k}^{+}\right)=(Q y)\left(t_{k}^{+}\right)= \\
& =L_{k} x_{k}+\int_{\alpha}^{t_{k}} L_{k} W_{1}\left(t_{k}, s\right) f(s, y(s)) d s+\sum_{\alpha \leq t_{j} \leq t_{k}} L_{k} W_{1}\left(t_{k}, t_{j}\right) \cdot h_{j}\left(y_{j}\right)- \\
& -\int_{t_{k}}^{\infty} L_{k} W_{2}\left(t_{k}, s\right) f(s, y(s)) d s-\sum_{j=k+1}^{\infty} L_{k} W_{2}\left(t_{k}, t_{j}\right) h_{j}\left(y_{j}\right)= \\
& =L_{k}\left[y_{k}+W_{1}\left(t_{k}, t_{k}\right) h_{k}\left(y_{k}\right)+W_{2}\left(t_{k}, t_{k}\right) h_{k}\left(y_{k}\right)\right]= \\
& =L_{k} y_{k}+H_{k} y_{k}
\end{aligned}
$$
estimate

Finally, as a consequence of the definition of the operator $Q$ we obtain the

$$
\|y(t)-x(t)\|=\|(Q y)(t)-x(t)\| \leq F(t) \quad(\alpha \leq t<\infty)
$$

which implies all assertions of Theorem 1.

Remark 1. For $\operatorname{dim} X<\infty$ Theorem 1 still holds without condition 3 and the second part of condition 4 .

Theorem 2. Let a solution $y \in((3),(4))_{\bar{t}}$ exist satisfying the condition

$$
\begin{aligned}
& \int_{\bar{t}}^{t}\left\|W_{1}(t, s) f(s, y(s))\right\| d s+\sum_{i<i_{j}<t}\left\|W_{1}\left(t, t_{j}\right) h_{j}\left(y_{j}\right)\right\|+ \\
& \quad+\int_{t}^{\infty}\left\|W_{2}(t, s) f(s, y(s))\right\| d s+\sum_{t \leq t_{j}<\infty}\left\|W_{2}\left(t, t_{j}\right) h_{j}\left(y_{j}\right)\right\| \underset{t \rightarrow \infty}{\rightarrow} 0
\end{aligned}
$$

Then there exists a solution $x \in((1),(2))_{i}$ for which $x(t)-y(t) \underset{t \rightarrow \infty}{\rightarrow} 0$. If for some $p \in[1, \infty]$ the left-hand side of relation (9) belongs to the space $L_{p}(\bar{t}, \infty)$, then $\|x(t)-y(t)\| \in L_{p}(\vec{t}, \infty)$ too.

Proof: For $t \geq \bar{t}$ define the function $x$ by means of the formula

$$
\begin{aligned}
x(t)=y(t)-\int_{t}^{t} W_{1}(t, s) f(s, y(s)) d s & -\sum_{i<t_{j}<t} W_{1}\left(t, t_{j}\right) h_{j}\left(y_{j}\right)+ \\
& +\int_{t}^{\infty} W_{2}(t, s) f(s, y(s)) d s+\sum_{t \leq t_{j}<\infty} W_{2}\left(t, t_{j}\right) h_{j}\left(y_{j}\right)
\end{aligned}
$$

By a straightforward verification, analogously to (7) and (8), it is established that $x \in((1),(2))_{t}$ and has the necessary properties.

Theorems 1 and 2 immediately imply the following theorem of asymptotic equivalence of impulsive equations (1), (2) and (3), (4): 
Theorem 3. Let for any $R>0$ the following conditions hold:

I.

$$
\int_{i}^{t} \sup _{\|z\| \leq R}\left\|W_{1}(t, s) f(s, z)\right\| d s+\sum_{i<t_{j}<t} \sup _{\|z\| \leq R}\left\|W_{1}\left(t, t_{j}\right) h_{j}(z)\right\|+
$$

$+\sup _{i \leq \tau \leq i}\left(\int_{t}^{\infty} \sup _{\|z\| \leq R}\left\|W_{2}(\tau, s) f(s, z)\right\| d s+\sum_{t \leq t_{j}<\infty} \sup _{\|z\| \leq R}\left\|W_{2}\left(\tau, t_{j}\right) h_{j}(z)\right\|\right] \rightarrow \underset{i \rightarrow \infty}{\rightarrow} 0$

2. The set $f\{(t, z): \bar{t} \leq t<\infty,\|z\| \leq R\}$ is relatively compact in $X$.

3. The mappings $H_{j}(j=1,2,3, \ldots)$ are continuous and the set $\bigcup_{j=1}^{\infty} h_{j}\{z \in X:\|z\| \leq R\}$ is relatively compact in $X$.

Then the impulsive equations (1), (2) and (8), (4) are asymptotically equivalent. Moteover, if the left-hand side of relation (10) belongs for some $p \in[1, \infty]$ to the space $L_{p}(\bar{t}, \infty)$, then $\|y(t)-x(t)\| \in L_{p}(\alpha, \infty)$ as well.

Remark 2. For $\operatorname{dim} X<\infty$ Theorem 3 still holds without condition 2 and the second part of condition 3 .

\section{References}

1. Bainov D.D., Kostadinov S.I. And Myshis A.D., Bounded and periodic solutions of differential equations with impulse effect in a Banach space, Differential and integral equations 1, no. 2 (1988), 223-230.

2. Bainov D.D., zabreiko P.P. and Kostadinov S.I., Stability of the general exponent of nonlinear impulsive differential equations in a Banach space, International J. of theoretical physics 27, no, 3 (1988), 373-380.

3. BRoUWER, F., On the asymptotic relationships between solutions of two systems of ordinary differential equations, $J$. of differential equations 6 (1969), 527-543.

4. Dishliev A.B. AND BAINOV D.D., Sufficient conditions for absence of beating in systems of differential equations with impulses, Applicable Anal. 18 (1985), 67-73.

5. FUTAK J., Asymptotic and integral equivalence of functional and ordinary differential equations, Fasciculi Mathematici 15 (1984), 97-108.

6. HAS̆C̆AK AND S̆VEC M., Integral equivalence of two systems of differential equations, Czechoslovak Mathematical Journal 32 (107) (1982), 423-436.

7. HAŠC̆ AK A., Integral equivalence between a nonlinear system and its nonlinear perturbation, Math. Slovaca 34, no. 4 (1984), 393-404.

8. Hu, Schouchuan and Lakshmikantham V., Periodic boundary value problems for second order impulsive differential systems, To appear in $J$. of Nonlinear Analysis. 
9. Hu, Schouchuan, Lakshmikantham V., Existence and continuation resuits for impuisive differential equations, To appear.

10. Kulev G.K. AND BAINov D.D., Asymptotic stability of systems with impulses by the direct method of Lyapunov, Bull. Austral. Math. Soc. 38 (1988), 113-123.

11. KulEV G.K. AND BAINOV D.D., Second method of Lyapunov and comparison principle for systems with impulse effect, $J$. of Computational and Applied Mathematics 23 (1988), 305-321.

12. LAKSHMIKANTHAM V.AND LIU XINZHI, Stability for impulsive differential systems in terms of two measures, Appl. Math. Comp. (to appear).

13. LaKSHMIKANTHAM V. AND LIU XINZHI, On quasi-stability for impulsive differential systems, To appear in J. Nonlinear Analysis.

14. MARLIN J.A. AND STRUBLE R.A., Asymptotic equivalence of nonlinear systems, $J$. of differential equations 6 (1969), 578-596.

15. MLL'MAN V.D. AND MYShKis A.D., On the stability of motion in the presence of impulses, Sibirsk. Mat. J. 1 (1960), 223-237 (in Russian).

16. SimEonov P.S. AND BAINov D.D., Asymptotic equivalence of two systems of differential equations with impulse effect, Systems control letters $3(1983), 297-301$.

P.O. Box 45

1504 Sofia

BULGARIA

Rebut el 16 de Juny de 1989 